Published in final edited form as:

J Am Chem Soc. 2005 April 6; 127(13): 4632-4639.

\title{
Structure-Based Optimization of a Non- $\beta$-lactam Lead Results in Inhibitors That Do Not Up-Regulate $\beta$-Lactamase Expression in Cell Culture
}

\author{
Donatella Tondi $\dagger^{\dagger} \ddagger$, Federica Morandi $\dagger, \ddagger$, Richard Bonnet $§$, M. Paola Costi ${ }^{\star} \ddagger$, and Brian K. \\ Shoichet ${ }^{\star \dagger}$ \\ Contribution from the Department of Pharmaceutical Chemistry, University of California - San \\ Francisco, 600 16th Street San Francisco, California 94143-2240, Dipartimento di Scienze \\ Farmaceutiche, Università degli Studi di Modena e Reggio Emilia, Via Campi 183, 41100, Modena, \\ Italy, and Service de Bactériologie, Faculté de Médecine, Université d'Auvergne, 63001 Clermont- \\ Ferrand, France
}

\begin{abstract}
Bacterial expression of $\beta$-lactamases is the most widespread resistance mechanism to $\beta$-lactam antibiotics, such as penicillins and cephalosporins. There is a pressing need for novel, non- $\beta$-lactam inhibitors of these enzymes. One previously discovered novel inhibitor of the $\beta$-lactamase AmpC, compound $\mathbf{1}$, has several favorable properties: it is chemically dissimilar to $\beta$-lactams and is a noncovalent, competitive inhibitor of the enzyme. However, at $26 \mu \mathrm{M}$ its activity is modest. Using the X-ray structure of the AmpC/1 complex as a template, 14 analogues were designed and synthesized. The most active of these, compound 10, had a $K_{i}$ of $1 \mu \mathrm{M}$, 26-fold better than the lead. To understand the origins of this improved activity, the structures of AmpC in complex with compound 10 and an analogue, compound 11, were determined by X-ray crystallography to 1.97 and $1.96 \AA$, respectively. Compound $\mathbf{1 0}$ was active in cell culture, reversing resistance to the third generation cephalosporin ceftazidime in bacterial pathogens expressing AmpC. In contrast to $\beta$ lactam-based inhibitors clavulanate and cefoxitin, compound $\mathbf{1 0}$ did not up-regulate $\beta$-lactamase expression in cell culture but simply inhibited the enzyme expressed by the resistant bacteria. Its escape from this resistance mechanism derives from its dissimilarity to $\beta$-lactam antibiotics.
\end{abstract}

\section{Introduction}

Microbial resistance to antibiotics is now a serious threat to public health. ${ }^{1,2}$ A pressing problem is resistance to the $\beta$-lactam antibiotics, including the penicillins and cephalosporins, which are among the most widely used class of antibiotics. Several mechanisms contribute to this resistance, including mutations in the target of these drugs, cell-wall biosynthesis transamidases called penicillin binding proteins, deletion and modification of the porin channels through which the drugs diffuse, and expression of pumps that export the drugs out of the bacterial cells. ${ }^{3-6}$ The most widespread resistance mechanism remains the expression of $\beta$-lactamase enzymes, which hydrolyze the lactam bond in the eponymous $\beta$-lactam ring of these drugs, inactivating them. 7,8

\footnotetext{
E-mail:costimp@unimore.it;shoichet@cgl.ucsf.edu.

† University of California, San Francisco.

†Università degli Studi di Modena e Reggio Emilia.

§Universitè d'Auvergne.a
}

Supporting Information Available: Detailed information related to the synthesis and chemical characterization of compounds 2-14 and a table listing all compounds characterized through ${ }^{1} \mathrm{H}$ NMR and their numbering (PDF). This material is available free of charge via the Internet at http://pubs.acs.org. 
To overcome these resistance enzymes, $\beta$-lactam molecules that inhibit (e.g. clavulanic acid) or evade (e.g. aztreonam) $\beta$-lactamases have been introduced. These molecules are themselves $\beta$-lactams and, like the penicillins, most are derivatives of microbial natural products that have been in the biosphere over evolutionary time. Consequently, resistance to them has evolved rapidly, often in the $\beta$-lactamases themselves. ${ }^{9}$ Mutant enzymes have arisen that can evade $\beta$ lactam-based $\beta$-lactamase inhibitors. ${ }^{10-12}$ Enzymes that are naturally resistant to current inhibitors, including class $C \beta$-lactamases such as AmpC, have become prominent in clinical settings. ${ }^{13}$ Mechanisms that alter the expression levels of the enzymes in the presence of the drugs or the inhibitors have appeared. There are strains of pathogenic bacteria that, recognizing the presence of a $\beta$-lactam-based inhibitor, will overexpress the $\beta$-lactamase that these drugs are meant to inhibit. ${ }^{14}$ There is thus a pressing need for novel $\beta$-lactamase inhibitors, not based on a $\beta$-lactam core structure. Such inhibitors would not be hydrolyzed by $\beta$-lactamases or mutant $\beta$-lactamases and would not be recognized by the suite of bacterial resistance mechanisms mobilized against $\beta$-lactams. ${ }^{15}$

Recently, we reported the structure-based discovery of a novel, noncovalent inhibitor of the widespread class C $\beta$-lac-tamase AmpC, compound 1 (Figure 1). ${ }^{16}$ This compound is dissimilar to penicillins and cephalosporins and binds to the enzymes noncovalently and reversibly, in contrast to the $\beta$-lactam substrates and inhibitors. Despite these differences, the $\mathrm{X}$-ray crystal structure of the AmpC/ $\mathbf{1}$ complex revealed that $\mathbf{1}$ complements the core of the active site, interacting with key residues involved in $\beta$-lactam recognition and hydrolysis such as Ser64, Lys67, Asn152, and Tyr221. We concluded that the ligand recognition encoded by the AmpC structure was plastic enough to accommodate inhibitors genuinely dissimilar to $\beta$ lactams, allowing a new departure in the medicinal chemistry of their inhibitors.

The very novelty of this inhibitor posed several problems, ones that are perhaps shared by many genuinely new leads. First, the inhibition constant of compound $\mathbf{1}$ was, at $26 \mu \mathrm{M}$, weak, and its activity in vivo was poor. Moreover, its novelty confronted us with an unanticipated design problem. Whereas one can draw upon 60 years of medicinal chemistry in designing analogues of $\beta$-lactams, ${ }^{17}$ something we ourselves have done in the past, ${ }^{18,19}$ in a novel series this course is unavailable. Second, whereas $\beta$-lactams are evolved for cellular efficacy, neither the purely synthetic compound $\mathbf{1}$ nor its analogues need be active against bacteria. Having discovered a novel lead, the question became, could we improve its affinity and biological activity?

Here we describe our preliminary efforts to improve this series of inhibitors. We continue to use a structure-based approach. In the structure of the AmpC/1 complex, the inhibitor complements the core of the active site but leaves a distal region open. We sought derivatives to take advantage of this region that were relatively easy to synthesize and would not diminish the solubility and "leadlike" properties of the inhibitors. ${ }^{20}$ We therefore focused on derivates that made new interactions with polar residues in the distal part of the AmpC site, including $\operatorname{Arg} 204$, or that tested features of the ligands that appeared important for binding. Fourteen analogues were synthesized, the best of which bound to AmpC with 26-fold improved affinity over 1. To understand their improved affinity, X-ray crystal structures were determined for two of the new analogues in complex with AmpC. To explore their biological potential, several of the new inhibitors were tested in bacterial cell culture against clinical pathogens. We observe intriguing differences in their biological effects on resistant bacteria compared to classic, $\beta$ lactam-based inhibitors such as clavulanate and cefoxitin.

\section{Results Synthesis.}

The synthesis of the methyl ester intermediates and their corresponding acids was accomplished by reacting the sulfonyl chloride with the appropriate amine in anhydrous 
pyridine (Scheme 1). The only exception was in the synthesis of compound $\mathbf{4 A}$, where the amine reacted with the monomethylphthalate in the presence of diisopropylamine (DIEA) and benzotriazol-1-yl-oxytripyrrolidinophosphonium hexafluoro-phosphate (PyBOP) to afford the desired methyl ester (Scheme 2). Each ester was subsequently hydrolyzed by suspending it in sodium hydroxide and isolating the final acids through precipitation with dilute $\mathrm{HCl}$.

All intermediate esters $(\mathbf{1 A - 1 4 A})$ were characterized either through elementary analysis or monodimensional ${ }^{1} \mathrm{H}$ NMR, or both, whereas all final carboxylic acids (1-14) were characterized through monodimensional ${ }^{1} \mathrm{H}$ NMR and either mass spectroscopy or elementary analysis, or all three (see Materials and Methods and Supporting Information, Table S1). The structures of all compounds were consistent with their analytical and spectroscopic data.

\section{Design, Enzymology, and Binding Affinity.}

We wished to investigate which groups in the lead inhibitor 1 were critical for binding, and should be maintained, and where we could improve affinity through chemical elaboration. We first focused on the 3-(sulfonylamino)-2-carboxylic acid thiophene moiety of 1 (Figure 1), which in the AmpC complex structure hydrogen bonds with active site residues. For instance, the carboxylate of $\mathbf{1}$ binds in the "oxyanion" hole of AmpC, hydrogen bonding with main chain amide nitrogens, Ser64, and a conserved water molecule (Wat401), while the sulfonamide hydrogen bonds with the conserved Asn 152 of AmpC. Derivates that replaced the thiophene carboxylate with a methyl ester substantially reduced affinity, consistent with the importance of the ion-dipole interactions made by this carboxylate (e.g., compound 1A, Table 1).

Similarly, inverting the order of the sulfonyl-amino group reduced affinity 10-20-fold (compare compounds $\mathbf{1}$ and $\mathbf{2}$ or $\mathbf{1 1}$ and 14, Table 1), and replacing the sulfonamide with an amide reduced affinity 8-fold, consistent with the importance of the hydrogen bond between this group and Asn152 observed crystallographically. We also replaced the thiophene of $\mathbf{1}$ with a more synthetically tractable benzene. This substitution diminished activity, but by less than 2-fold (compound 4, Table 1).

In an attempt to increase the affinity of compound $\mathbf{1}$, we considered derivates that improved either the apolar or polar complementarity in the distal chloro-phenyl ring of the inhibitor. This functionality is located in a more open region of the active site, flanked by the conserved Tyr221 on the "floor" of the active site, bulk solvent at the "top" of the inhibitor, and polar and apolar residues such as Arg204, Gly320, Thr319, and Val211 to its side. Introducing bulk or hydrophobicity, such as in 5, 6, and 13, affected affinity only modestly. More polar derivates, such as carboxylate and hydroxyl groups, improved affinity more substantially, up to 26-fold in compounds 7-10 (Table 1).

\section{X-ray Crystallographic Structure Determination.}

To investigate the structural bases for the higher affinities found for the new series, we determined the crystal structure of AmpC in complex with compound $\mathbf{1 0}$ and with compound 11 at 1.97 and 1.96 å resolution, respectively (Table 2). Proline and glycine residues excluded, $92.2 \%$ of the amino acids were in the most favored regions of the Ramachandran plot and $7.8 \%$ were in the additionally allowed regions for the crystal structure of AmpC/10. For AmpC/11, $92.5 \%$ of the nonproline and nonglycine amino acids were in the most favored regions of the Ramachandran plot and $7.5 \%$ were in the additionally allowed regions. The positions of both inhibitors in the active sites of each structure were unambiguously identified in the initial $F_{\mathrm{O}^{-}}$ $F_{\mathrm{c}}$ difference map contoured at $3 \sigma$. In the AmpC/10 complex, the inhibitor was observed and modeled in both monomers in the asymmetric unit, whereas in the AmpC/11 complex, the inhibitor was only observed and modeled in one of the two monomers. 
Overall, the complexes with $\mathbf{1 0}$ and $\mathbf{1 1}$ resemble that of the AmpC/1 complex, with a few interesting differences. Key hydrogen bond interactions in the conserved parts of all three structures, the thiophene-carboxylate and the sulfonamide functionalities, are maintained (Tables S2 and S3, Supporting Information; Figures 2 and 3). The thiophene carboxylate in both 10 and 11 hydrogen bonds with both the $\mathrm{O} \gamma$ and the main-chain $\mathrm{NH}$ of the hydrolytic Ser64, with a third hydrogen bond formed with the main-chain nitrogen of Ala318 and a fourth with the conserved Wat401. There are two enzyme monomers in the asymmetric unit in the AmpC structures, and in one of them, monomer 2, a fifth hydrogen bonds is formed to Wat 481 . As with the lead compound $\mathbf{1}$, the thiophene ring is within van der Waals distance of residues Leu119 and Leu293, which form a hydrophobic patch on AmpC. A sulfonamide oxygen

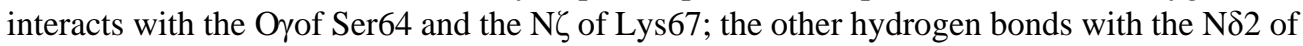
Asn 152. As in the AmpC/1 complex, the nitrogen atom of the sulfonamide interacts with the main-chain oxygen of Ala318.

Differences emerge in the 4'-carboxylate-2'-hydroxy benzene ring, which is the point of substitution for compound 10. The ring itself appears to form edge-to-face quadrupole interactions with Tyr221 via the introduced 2'-hydroxyl of 10; the distance between this hydroxyl and C $\delta 1$ of Tyr221 is $3.43 \AA$. This interaction is also present in the structure of the lead 1, but in this case it appears to be a ring-ring or quadrupolar interaction, whereas with 10 the intercession of the hydroxyl makes it a dipole-quadrupolar interaction. The presence of the 2'-hydroxyl also displaces $\mathbf{1 0}$ slightly "up" in the site, moving the center of the ring away from Tyr221 and toward polar residues such Arg204 and Thr319, by about $0.2 \AA$ in monomer 2. In both crystallographic monomers 1 and 2 of AmpC the 4'-carboxylate points toward the solvent accessible entry of the binding site. In monomer 1 , this carboxylate hydrogen bonds with an ordered water molecule, Wat521 (distance $2.9 \AA$ ), which in turn hydrogen bonds with the N $\varepsilon$ of Arg204 (distance, $2.8 \AA$ ). In monomer 2, Arg204 adopts a different conformation and these interactions are not observed. Instead, a new interaction is observed between $G \ln 120$ and the sulfonamide of $\mathbf{1 0}$, with the Gln120 in this monomer pointing toward the inhibitors rather than out of the site, as it does in monomer 1. Also in monomer 2, difference electron density suggests that a second conformation of the inhibitor may exist, with the distal phenyl ring rotated by $180^{\circ}$, allowing the $2^{\prime}$-hydroxyl group to hydrogen bond with the side chain of Asn343 through an ordered water molecule.

To investigate how important these polar and quadrupolar interactions might be, we also determined the structure of the AmpC/11 complex by X-ray crystallography. Compound $\mathbf{1 1}$ replaces the 4'-carboxylate of $\mathbf{1 0}$ with a 3'-nitro and correspondingly loses an order of magnitude in affinity (Table 1). In the structure of the AmpC/11 complex, the inhibitor is moved slightly down in the site, keeping the quadrupole edge-to-face stacking with Tyr221, but this time without the intercession of the 2'-hydroxyl, which is no longer present. The 3 '-nitro is relatively distant from Arg204, and we observe no interaction with an ordered water; the loss of these polar interactions may be consistent with the diminished affinity for this inhibitor.

\section{Microbiology.}

To investigate the potential of these compounds to reverse antibiotic resistance, we undertook antimicrobial activity studies in bacterial cell culture. Compounds $\mathbf{1 0}$ and $\mathbf{1 1}$ were tested for synergy with the third generation cephalosporin ceftazidime (CAZ) against pathogenic bacteria from clinical isolates from the Clermont-Ferrand University Hospital (France), and the results were compared with those of the lead compound $\mathbf{1}$. Strains tested were E. aerogenes 298, E. cloacae P99, C. freundii 63, K. pneumoniae Kus, K. pneumoniae 704, and P aeruginosa CF34. All were resistant to CAZ because of the expression of class $C \beta$-lactamases. The minimum inhibitory concentration (MIC) of CAZ against these pathogens ranged from 256 to $64 \mu \mathrm{g} / \mathrm{mL}$ (Table 3; Figure 4). 
Compound 10 increased bacterial susceptibility to CAZ, improving its MIC values by 4- to 8fold. This was sufficient to partially restore clinically relevant susceptibility for $E$.

Aerogenes 298, E. cloacae P99, C. freundii 63, and K. pneumoniae 704. Conversely, the lead compound $\mathbf{1}$ and compound 11, both of which have lower affinities than does $\mathbf{1 0}$, had relatively little effect on the potency of CAZ. Consistent with this result, $\mathbf{1 0}$ synergized the activity of $\mathrm{CAZ}$ and the penicillin piperacillin (PIP) in disk diffusion assays, as can be seen by the characteristic tear-drop shapes of the zones of bacterial growth inhibition (Figure 5). This contrasts dramatically with the same experiment performed with the $\beta$-lactam-based inhibitors clavulanate and cefoxitin ( $\mathrm{IC}_{50}$ of $340 \mathrm{nM}$ vs $\mathrm{AmpC}$; not shown), both of which induce AmpC expression. $15,21,22$ Although clavulanate and cefoxitin inhibit the AmpC- type $\beta$-lactamase expressed by these cells, they nevertheless antagonize the activity of CAZ and PIP in the disk diffusion studies. This results in flattened zones of bacterial grown inhibition, reflecting the up-regulation of $\beta$-lactamase production in the E. cloacae bacteria.

\section{Discussion}

There is a pressing need for novel inhibitors to combat antibiotic resistance, but the discovery of such confronts one with new problems. In the case of lead compound 1 , a non- $\beta$-lactam inhibitor of $\beta$-lactamases, the challenges were to improve the binding affinity in a series where one could not simply apply 60 years worth of $\beta$-lactam structure-activity relationships and to improve the biological efficacy in a scaffold that, unlike $\beta$-lactams, had not been evolved for in vivo activity. Three important results emerge from our preliminary efforts to improve this series of compounds. First, a cycle of structure-based design and testing led to small, soluble inhibitors whose improved affinities could be explained on the basis of the X-ray structures subsequently determined for their complexes. Second, the improved affinity is mirrored by the improved activity in cell culture. Third, new biology follows from the new chemical structures in this series, which, not being $\beta$-lactams, simply inhibit $\beta$-lactamase and do not up-regulate its expression in cell culture, as do classic $\beta$-lactam-based inhibitors such as clavulanate (Figure $5) .7$

It is encouraging that modest changes in this series can improve inhibition significantly (compare the lead $\mathbf{1}$ with 10, Table 1). Also, the resulting analogues remain relatively soluble and small, leaving further room for chemical elaboration. Indeed, an encouraging aspect of this series is that increased affinity comes from polar groups that act to maintain the overall solubility of the compounds, most particularly the addition of a carboxylate on the distal phenyl ring. Exactly what interactions are responsible for the improved affinity is less certain. The crystal structures of the $1.0 \mu \mathrm{M}$ inhibitor 10 and the $14 \mu \mathrm{M}$ inhibitor 11 in complex with AmpC suggest that water-mediated interactions with Arg204 are responsible for the improved affinity of 10. This conclusion must be seen as quite tentative at this point, since this interaction is only seen in one of the two monomers of the AmpC/10 complex. From a molecular recognition standpoint, it is surprising that a water-mediated hydrogen bond between the carboxylate and Arg204, in a relatively solvent-exposed region, can significantly improve affinity, though this sort of effect is not unprecedented in AmpC. ${ }^{23}$ Other explanations are possible; for instance the improved affinity may derive from an overall improved complementarity to the highly electropositive active site of AmpC. Detailed conclusions as to the importance of these interactions must remain tentative pending more rigorous analyses, such as double-perturbation thermodynamic cycles; ${ }^{23,24}$ what we can say is that the X-ray structures of these complexes provide templates for designing and understanding such experiments.

What might justify this effort is that the improved enzyme affinity of inhibitor $\mathbf{1 0}$ results in 4to 8-fold improvement in antibiotic efficacy. In bacterial cell culture, a 1:1 ration of $\mathbf{1 0}$ to CAZ synergizes the activity of the latter against widespread hospital pathogens such as E. cloacae and $C$. freundii (Table 3 and Figure 4) and is significantly more potent than the lead compound 
1 or compound 11. This antibiotic effect suggests that this series of inhibitors, whose $K_{\mathrm{i}}$ values remain relatively modest, may be useful leads for further optimization. The X-ray crystal structures of compound $\mathbf{1 0}$ in complex with AmpC provide atomic resolution templates for future design efforts.

Perhaps the most striking result to emerge from this study, however, comes from comparing the effects of the classic $\beta$-lactam-based inhibitors clavulanate and cefoxitin with those of compound 10 on an inducible strain of E. cloacae. These bacteria up-regulate the expression of class $C \beta$-lactamase in the presence of $\beta$-lactams such as clavulanate, thereby overwhelming these $\beta$-lactams with the very enzyme they are meant to inhibit (Figure 5A,B). Conversely, compound 10, which is not a $\beta$-lactam, simply inhibits the $\beta$-lactamase expressed by these bacteria, it does not lead to its up-regulation. Thus, the novel chemical structures represented by this series of inhibitors leads to novel, therapeutically relevant biological effects.

\section{Materials and Methods}

\section{Chemistry.}

All reagents were purchased from Sigma-Aldrich or Fluka and were reagent grade. Progress of the reaction was monitored by thin-layer chromatography (TLC) on silica gel plates (Riedelde-Haen, Art. 37341). Merck silica gel (Kieselgel 60) was used for flash chromatography (230$400 \mathrm{mesh}$ ) when required. Extracts were dried over $\mathrm{MgSO}$, and solvents were removed under reduced pressure.

Melting points were determined on a Büchi 510 capillary melting point apparatus and are uncorrected. Elemental analyses were performed on a Perkin-Elmer Elemental Analyzer 240C. ${ }^{1} \mathrm{H}$ NMR spectra were recorded on a Bruker DPX $200 \mathrm{MHz}$ spectrometer (Centro Interdipar-timentale Grandi Strumenti Università di Modena) with trimethylsilane (TMS) as internal standard; the values of the chemical shifts $(\delta)$ are given in parts per million and coupling constants $(J)$ in hertz. Dimethyl sulfoxide- $d_{6}\left(\right.$ DMSO- $\left.d_{6}\right)$ was used as the solvent. Mass spectra were determined on a Finnigan MAT SSQ 710 A mass spectrometer (EI, $70 \mathrm{eV}$ ).

\section{3-((4-Chlorophenyl)sulfamoyl)thiophene-2-carboxylic Acid Methyl Ester (1A).}

The starting amine, 4-chlorophenylamine $(35 \mathrm{mg}, 0.28 \mathrm{mmol})$ was dissolved in $10 \mathrm{~mL}$ of anhydrous pyridine, and the 3-(chlorosulfonyl)thiophene-2-carboxylic acid methyl ester (100 $\mathrm{mg}, 0.42 \mathrm{mmol}$ ) was added dropwise to the solution under stirring. The reaction was stirred for $24 \mathrm{~h}$, at $50{ }^{\circ} \mathrm{C}$, under nitrogen. The resulting solution was treated with water and ice; the crude product, corresponding to the 3-((4-chlorophenyl)sulfamoyl)thiophene-2-carboxylic acid methyl ester (1A) was formed and isolated through filtration. Yield: $73 \mathrm{mg}, 80 \%$; $\mathrm{mp} 87-$ $90{ }^{\circ} \mathrm{C}$. Anal. Calcd for $\mathrm{C}_{12} \mathrm{H}_{10} \mathrm{ClNO}_{4} \mathrm{~S}_{2}: \mathrm{C}, 43.44 ; \mathrm{H}, 3.04 ; \mathrm{N}, 4.22$. Found: $\mathrm{C}, 43.51 ; \mathrm{H}, 3.10$; $\mathrm{N}, 4.28$.

\section{3-((4-Chlorophenyl)sulfamoyl)thiophene-2-carboxylic Acid (1).}

Compound $1 \mathrm{~A}$ (60 mg, $0.18 \mathrm{mmol}$ ) was suspended in $10 \mathrm{~mL}$ of $2 \mathrm{~N} \mathrm{NaOH}$, and the reaction was stirred for $1 \mathrm{~h}$ at $80^{\circ} \mathrm{C}$, until the starting material was completely dissolved. Thereafter, the solution was cooled, and dilute $\mathrm{HCl}$ was added until the solution reached $\mathrm{pH} 2$. A precipitate, corresponding to 3-((4-chlorophenyl)sulfamoyl)thiophene-2-carboxylic acid (1) was formed and isolated through filtration. Yield: $42 \mathrm{mg}, 73 \%$; mp 195-198 ${ }^{\circ} \mathrm{C} .{ }^{1} \mathrm{H}$ NMR: 7.97 (H1, d, J $5.4 \mathrm{~Hz}) ; 7.49(\mathrm{H} 2$, d, J $5.4 \mathrm{~Hz}) ; 7.21(\mathrm{H} 3, \mathrm{~d} / \mathrm{o}, \mathrm{J} 8.8 \mathrm{~Hz}) ; 7.40(\mathrm{H} 4, \mathrm{~d} / \mathrm{o}, \mathrm{J} 9 \mathrm{~Hz}) ; 7.40(\mathrm{H} 5, \mathrm{~d} /$ o, J $9 \mathrm{~Hz}) ; 7.21(\mathrm{H6}, \mathrm{d} / \mathrm{o}, \mathrm{J} 8.8 \mathrm{~Hz}) ; 10.20(\mathrm{H} 7, \mathrm{~s})$. Anal. Calcd for $\mathrm{C}_{11} \mathrm{H}_{8} \mathrm{ClNO}_{4} \mathrm{~S}_{2}: \mathrm{C}, 41.58$; H, 2.54; N, 4.41. Found: C, 41.45; H, 2.48; N,4.51. 
Intermediate ester derivatives (2A-14A) and final carboxylic acids (2-14) were synthesized according to the method followed for $\mathbf{1 A}$ and 1.Details are reported in the Supporting Information.

\section{Enzymology.}

Inhibitors were dissolved in DMSO at a concentration of $50 \mathrm{mM}$; more dilute stocks were subsequently prepared as necessary. Kinetic measurements were performed using nitrocefin as substrate in $50 \mathrm{mM}$ Tris buffer, $\mathrm{pH} 7.0$, and monitored in an HP8453 UV-vis spectrophotometer. The $K_{\mathrm{m}}$ of nitrocefin for AmpC in this buffer was $127 \mu \mathrm{M}$. The concentration of AmpC was determined spectrophotometrically in concentrated stock solutions made from lyophilized powder and subsequently diluted; this enzyme had been previously expressed and purified, as described. ${ }^{15}$ The concentration of enzyme in all reactions was 1.75 $\mathrm{nM}$. Inhibition $K_{\mathrm{i}}$ values were obtained from $\mathrm{IC}_{50}$ plots assuming competitive inhibition, an assumption consistent with both previous inhibition patterns in this series ${ }^{16}$ and with experiments investigating the effect of increasing substrate concentrations (not shown). For compound 10 , the $K_{\mathrm{i}}$ value was also obtained by comparison of progress curves in the presence and in the absence of inhibitor; ${ }^{18,25,26}$ the result was consistent with the value determined from the $\mathrm{IC}_{50}$ plots.

\section{Crystal Growth and Structure Determination.}

Cocrystals of AmpC in complex with compounds $\mathbf{1 0}$ and $\mathbf{1 1}$ were grown by vapor diffusion in hanging drops equilibrated over $1.8 \mathrm{M}$ potassium phosphate buffer ( $\mathrm{pH}$ 8.7) using microseeding techniques. The initial concentration of the protein in the drop was $3.8 \mathrm{mg} / \mathrm{mL}$, and the concentrations of compounds $\mathbf{1 0}$ and $\mathbf{1 1}$ were $0.8 \mathrm{mM}$. The compounds were added to the crystallization drops in a $1.2 \%$ DMSO, $1 \mathrm{M}$ potassium phosphate buffer $(\mathrm{pH} 8.7)$ solution. Crystals appeared a few weeks after equilibration at $21^{\circ} \mathrm{C}$. Before data collection, crystals were immersed in a cryoprotectant solution of $20 \%$ sucrose, $1.8 \mathrm{M}$ potassium phosphate, $\mathrm{pH}$ 8.7 , for about $30 \mathrm{~s}$, and were flash-cooled in liquid nitrogen.

Diffraction data were collected on frozen crystals at the Advance Light Source (ALS, Lawrence Berkeley Laboratory, CA). Both data sets were measured from single crystals. Reflections were indexed, integrated, and scaled using the HKL software package. ${ }^{27}$ For both structures, the space group was $C_{2}$, with two molecules in the asymmetric unit. For AmpC/10, molecule 1 of the asymmetric unit was modeled with 352 residues, including the inhibitor ( 7 residues, 284290, were left out due to poor density in this region, which is often disordered in AmpC structures), and molecule 2 was modeled with 359 residues, including the inhibitor. For $\mathrm{AmpC} /$ 11, molecule 1 was modeled with 342 residues (16 residues were left out), and molecule 2 of the asymmetric unit was modeled 359 residues, including the inhibitor. The initial phasing models were an apo AmpC structure (PDB entry 1KE4) for AmpC/10 and an AmpC/boronic acid complex (PDB entry 1MY8) for AmpC/11, with inhibitor, water molecules, and ions removed. The models were positioned using rigid body refinement and refined using the maximum likelihood target in CNS including: simulated annealing, positional minimization, and individual $B$-factor refinement, with a bulk solvent correction. ${ }^{28} \sigma A$-weighted electron density maps were calculated using CNS and used in further steps of manual model rebuilding and placement of water molecules with the programs XtalView and O. ${ }^{29,30}$ The inhibitors were built into the $2 F_{\mathrm{o}}-F_{\mathrm{c}}$ and $F_{\mathrm{O}}-F_{\mathrm{c}}$ electron density maps in each active site of the asymmetric unit. Subsequent refinement cycles consisted of positional minimization and $B-$ factor refinement in CNS.

\section{Microbiology.}

Compounds $\mathbf{1 0}$ and $\mathbf{1 1}$ were tested for synergy with the $\beta$-lactam ceftazidime against pathogenic bacteria from clinical isolates at the Clermont-Ferrant Hospital (France); these 
bacteria were resistant to $\beta$-lactams because of the expression of class $C \beta$-lactamases. Strains of bacteria tested were E. aerogenes 298 , E. cloacae $\mathrm{P} 99, C$. freundii $63, K$. pneumoniae Kus, K. pneumoniae 704, and P. aeruginosa CF34. ${ }^{31-33}$ Minimum inhibitor concentration values were determined with Mueller-Hinton broth using the microdilution method according to NCCLS guidelines. ${ }^{34}$ To test the inhibitory activity, compounds were dissolved in DMSO and dilutions were performed using growth medium. An adequate final concentration in which to determine the MIC was obtained where the concentration of DMSO was $<1 \%$. The ratio Inhib/CAZ was 1 . A control done with DMSO showed that DMSO did not have an effect in the bacteria growth.

For the $\beta$-lactamase induction experiments, plates of Mueller-Hinton agar were inoculated with a clinical strain of E. cloacae in which production of AmpC is inducible by $\beta$-lactams (Clermont-Ferrand hospital, unpublished strain). Inhibitors were added to blank disks, and the final content of inhibitor per disk was $128 \mu \mathrm{g}$ for compound $\mathbf{1 0}$ and $8 \mu \mathrm{g}$ for clavulanic acid. Disks of ceftazidime, cefoxitin, and piperacillin contained 30,30 , and $75 \mu \mathrm{g}$, respectively. ${ }^{35}$

\section{Data Deposition.}

The coordinates and structure factors have been deposited in the Protein Data Bank under accession codes $1 \mathrm{XGJ}$ for AmpC in complex with compound $\mathbf{1 0}$ and under accession code 1XGI for AmpC in complex with 11.

\section{Acknowledgements}

This work was supported by Grant GM63815 (to B.K.S.). We thank Piergiorgio Pecorari for his valuable suggestions and Alberto Venturelli for conversations, Yu Chen and Veena Thomas for reading this manuscript, and Jesus Blazquez for introducing some of us to synergy and antagonism tests for $\beta$-lactamase inhibitors.

\section{References}

1. Rice LB, Bonomo RA. beta-Lactamases: Which Ones Are Clinically Important? Drug Resist Updates 2000;3(3):178-189.

2. Cosgrove S, Carmeli Y. The Impact of Antimicrobial Resistance on Health and Economic Outcomes. Clin Infect Dis 2003;36:1433-1437. [PubMed: 12766839]

3. Chambers HF. Penicillin-Binding Protein-Mediated Resistance in Pneu-mococci and Staphylococci. J Infect Dis 1999;179(Suppl 2):353-359.

4. Li XZ, Nikaido H. Efflux-Mediated Drug Resistance in Bacteria. Drugs 2004;64(2):159-204. [PubMed: 14717618]

5. Li XZ, Zhang L, Nikaido H. Efflux Pump-Mediated Intrinsic Drug Resistance in Mycobacterium smegmatis. Antimicrob Agents Chemother 2004;48(7):2415-2423. [PubMed: 15215089]

6. Thiolas A, Bornet C, Davin-Regli A, Pages JM, Bollet C. Resistance to Imipenem, Cefepime, and Cefpirome Associated with Mutation in Omp36 Osmoporin of Enterobacter Aerogenes. Biochem Biophys Res Commun 2004;317(3):851-856. [PubMed: 15081418]

7. Bennett PM, Chopra I. Molecular Basis of beta-Lactamase Induction in Bacteria. Antimicrob Agents Chemother 1993;37(2):153-158. [PubMed: 8452343]

8. Essack SY. The Development of beta-Lactam Antibiotics in Response to the Evolution of betaLactamases. Pharm Res 2001;18(10):1391-1399. [PubMed: 11697463]

9. Medeiros AA. Cooperative Evolution of Mechanisms of beta-Lactam Resistance. Clin Microbiol Infect 2000;6(Suppl 3):27-33. [PubMed: 11449645]

10. Meroueh SO, Roblin P, Golemi D, Maveyraud L, Vakulenko SB, Zhang Y, Samama JP, Mobashery S. Molecular Dynamics at the Root of Expansion of Function in the M69L Inhibitor-Resistant TEM $\beta$-Lacta-mase from Escherichia coli. J Am Chem Soc 2002;124:9422-9430. [PubMed: 12167037]

11. Sun T, Bethel CR, Bonomo RA, Knox JR. Inhibitor-Resistant Class A $\beta$-Lactamases: Consequences of the Ser130-to-Gly Mutation Seen in Apo and Tazobactam Structures of the SHV-1 Variant. Biochemistry 2004;43:14111-14117. [PubMed: 15518561] 
12. Bush K. beta-Lactamases of Increasing Clinical Importance. Curr Pharm Des 1999;5(11):839-845. [PubMed: 10539991]

13. Philippon A, Arlet G, Jacoby G. Plasmid-Determined AmpC-Type beta-Lactamases. Antimicrob Agents Chemother 2002;46(1):1-11. [PubMed: 11751104]

14. Hanson ND, Sanders CC. Regulation of Inducible AmpC beta-Lactamase Expression among Enterobacteriaceae. Curr Pharm Des 1999;5(11):881-894. [PubMed: 10539994]

15. Powers RA, Blazquez J, Weston GS, Morosini MI, Baquero F, Shoichet BK. The Complexed Structure and Antimicrobial Activity of a Non-beta-lactam Inhibitor of AmpC beta-Lactamase. Protein Sci 1999;8(11):2330-2337. [PubMed: 10595535]

16. Powers RA, Morandi F, Shoichet BK. Structure-Based Discovery of a Novel, Noncovalent Inhibitor of AmpC beta-Lactamase. Structure (London) 2002;10(7):1013-1023.

17. Konaklieva MI. Lactams as Inhibitors of Serine Enzymes. Curr Med Chem: Anti-Infect Agents 2002;1 (3):215-238.

18. Caselli E, Powers RA, Blasczcak LC, Wu CY, Prati F, Shoichet BK. Energetic, Structural, and Antimicrobial Analyses of beta-Lactam Side Chain Recognition by beta-Lactamases. Chem Biol 2001;8(1):17-31. [PubMed: 11182316]

19. Trehan I, Morandi F, Blaszczak LC, Shoichet BK. Using Steric Hindrance To Design New Inhibitors of Class C beta-Lactamases. Chem Biol 2002;9(9):971-980. [PubMed: 12323371]

20. Oprea TI. Current Trends in Lead Discovery: Are We Looking for the Appropriate Properties? Mol Diversity 2002;5(4):199-208.

21. Kadima TA, Weiner JH. Mechanism of Suppression of Piperacillin Resistance in Enterobacteria by Tazobactam. Antimicrob Agents Chemother 1997;41(10):2177-2183. [PubMed: 9333044]

22. Sanders CC, Bradford PA, Sanders WEJ. Penicillin-Binding Proteins and Induction of AmpC $\beta$ Lactamase. Antimicrob Agents Chemother 1997;41:2013-2015. [PubMed: 9303404]

23. Roth T, Minasov G, Shoichet B. Thermodynamic Cycle Analysis and Inhibitor Design against $\beta$ Lactamase. Biochemistry 2003;42:14483-14491. [PubMed: 14661960]

24. Fersht AR, Shi JP, Knill-Jones J, Lowe DM, Wilkinson AJ, Blow DM, Brick P, Carter P, Waye MMY, Winter G. Hydrogen Bonding and Biological Specificity Analysed by Protein Engineering. Nature 1985;314:235. [PubMed: 3845322]

25. Waley GS. A Quick Method for the Determination of Inhibition Constants. Biochem J 1982;205:631633. [PubMed: 7150236]

26. Weston GS, Blazquez J, Baquero F, Shoichet BK. Structure-Based Enhancement of Boronic Acid Inhibitors of AmpC $\beta$-Lactamase. J Med Chem 1998;41:4577-4586. [PubMed: 9804697]

27. Otwinowski Z, Minor W. Processing of X-ray Diffraction Data Collected in Oscillation Mode. Methods Enzymol, Macromol Crystallogr, Part A 1997;276:307-326.

28. Brunger AT, Adams PD, Clore GM, DeLano WL, Gros P, Grosse-Kunstleve RW, Jiang JS, Kuszewski J, Nilges M, Pannu NS, Read RJ, Rice LM, Simonson T, Warren GL. Crystallography and NMR System: A New Software Suite for Macromolecular Structure Determination. Acta Crystallogr, Sect D: Biol Crystallogr 1998;54(Pt 5):905-921. [PubMed: 9757107]

29. McRee DE. XtalView/Xfit-A Versatile Program for Manipulating Atomic Coordinates and Electron Density. J Struct Biol 1999;125(2-3):156-165. [PubMed: 10222271]

30. Jones TA, Zou JY, Cowan SW, Kjeldgaard M. Improved Methods for Building Protein Models in Electron Density Maps and the Location of Errors in These Models. Acta Crystallogr, Sect A: Found Crystallogr 1991;47:110-119.

31. Bauernfeind A, Schneider I, Jungwirth R, Sahly H, Ullmann U. A Novel Type of AmpC betaLactamase, ACC-1, Produced by a Klebsiella Pneumoniae Strain Causing Nosocomial Pneumonia. Antimicrob Agents Chemother 1999;43(8):1924-1931. [PubMed: 10428914]

32. De Champs C, Poirel L, Bonnet R, Sirot D, Chanal C, Sirot J, Nordmann P. Prospective Survey of beta-Lactamases Produced by Ceftazi-dime-Resistant Pseudomonas aeruginosa Isolated in a French Hospital in 2000. Antimicrob Agents Chemother 2002;46(9):3031-3034. [PubMed: 12183264]

33. Fleming PC, Goldner M, Glass DG. Observations on the Nature, Distribution, and Significance of Cephalosporinase. Lancet 1963;29(1):1399-1401. [PubMed: 13945492] 
34. NCCLS, National Committee for Clinical Laboratory Standards. Methods for Dilution Antimicrobial Tests for Bacteria That Grow Aerobically Approved Standard, 4th ed.; NCCLS document M7-A5; NCCLS: Wayne, PA, 2000.

35. Jarlier V, Nicolas M, Fournier G, Philippon A. Extended Broad-Spectrum beta-Lactamases

Conferring Transferable Resistance to Newer beta-Lactam Agents in Enterobacteriaceae: Hospital Prevalence and Susceptibility Patterns. Rev Infect Dis 1988;10(4):867-878. [PubMed: 3263690] 


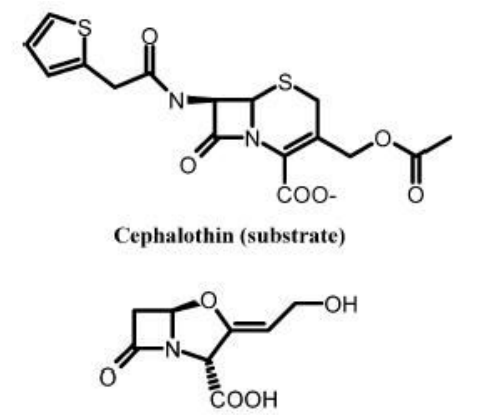

Clavulanic acid (inhibitor)

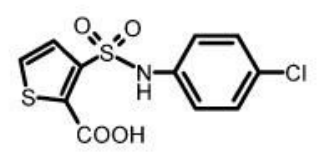

Lead compound 1 (Inhibitor)

Figure 1.

Characteristic $\beta$-lactam substrate and inhibitor of AmpC, and the lead compound for the novel inhibitor family discussed here. 
A
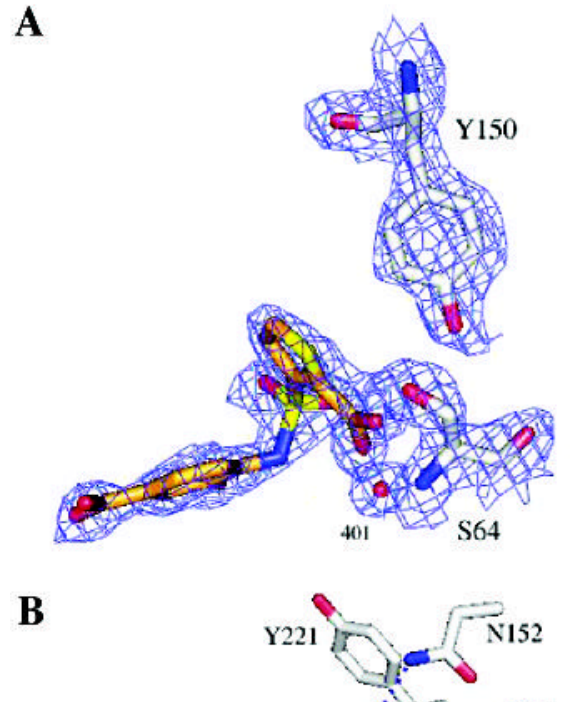

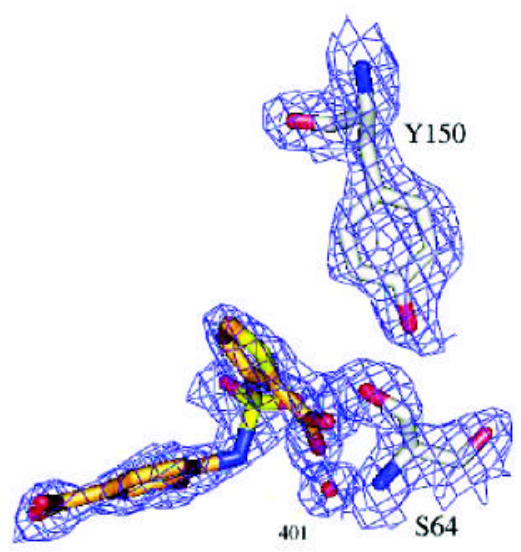

40

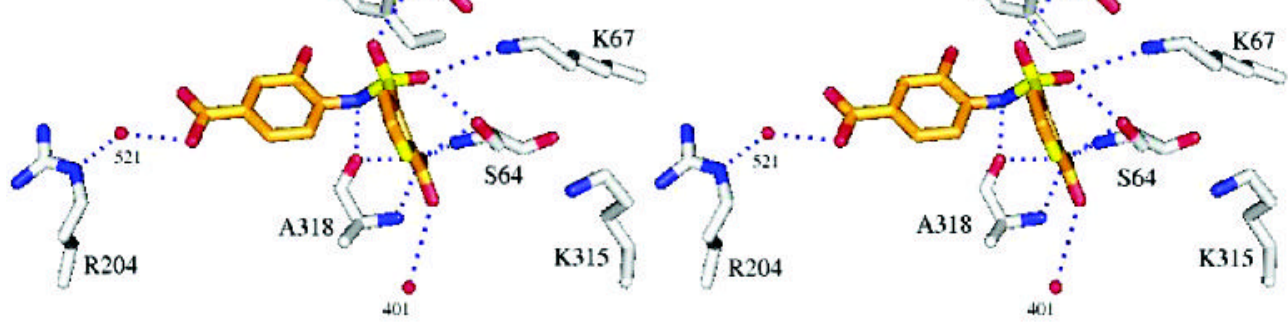

Figure 2.

$\mathrm{X}$-ray crystal structure of the AmpC/10 complex. (A) Stereoview of $2 F_{\mathrm{O}}-F_{\mathrm{c}}$ electron density maps contoured at $1 \sigma$ of the refined model. Carbon atoms are gray for the protein and orange for the ligand; oxygen atoms, red; nitrogen atoms, blue; sulfur atoms, yellow. (B) AmpC/10 complex in the active site. Dashed blue lines represent hydrogen bonds; red spheres represent water molecules. Interaction distances are listed in Table S2, Supporting Information. Figures 2 and 3 were generated with Pymol (http://pymol.sourceforge.net/). 
$\mathbf{A}$

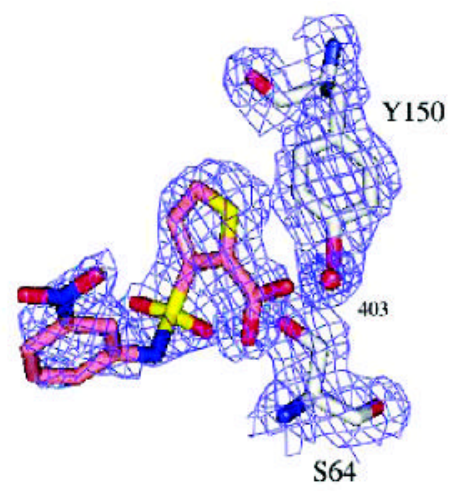

B

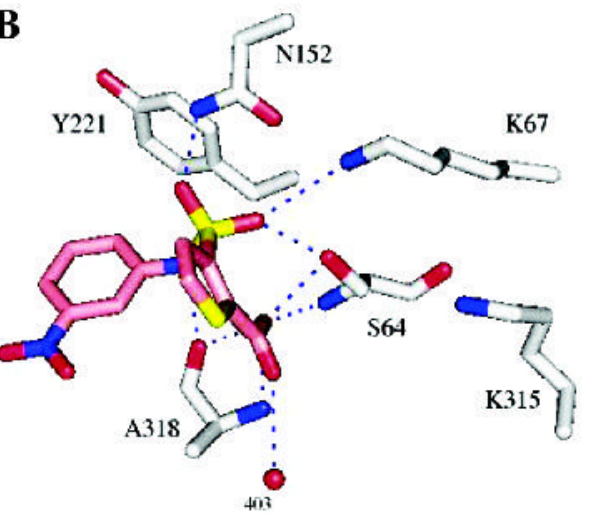

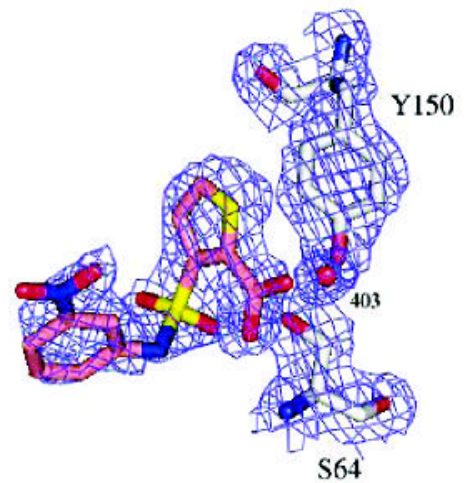

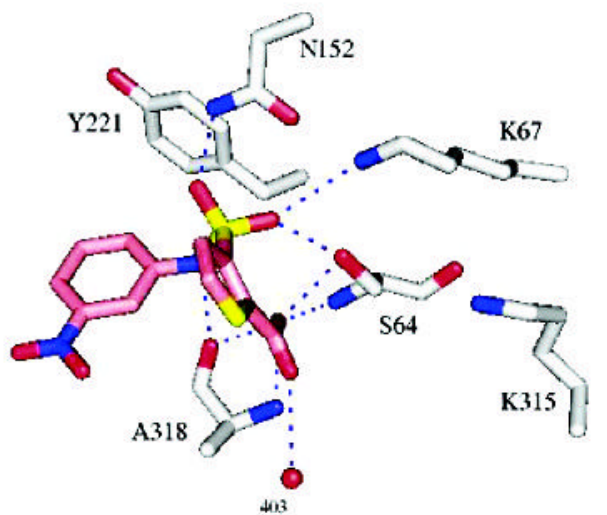

Figure 3.

$\mathrm{X}$-ray crystal structure of the AmpC/11 complex. (A) Stereoview of $2 F_{\mathrm{o}}-F_{\mathrm{c}}$ electron density maps (blue, contoured at $1 \sigma$ ) of the refined model. Carbon atoms are colored gray for the protein and pink for the ligand; oxygen atoms, red; nitrogen atoms, blue; sulfur atoms, yellow. (B) AmpC/11 complex in the active site. Dashed blue lines represent key hydrogen bonds. Red spheres represent water molecules. Interaction distances are listed in Table S3, Supporting Information. 


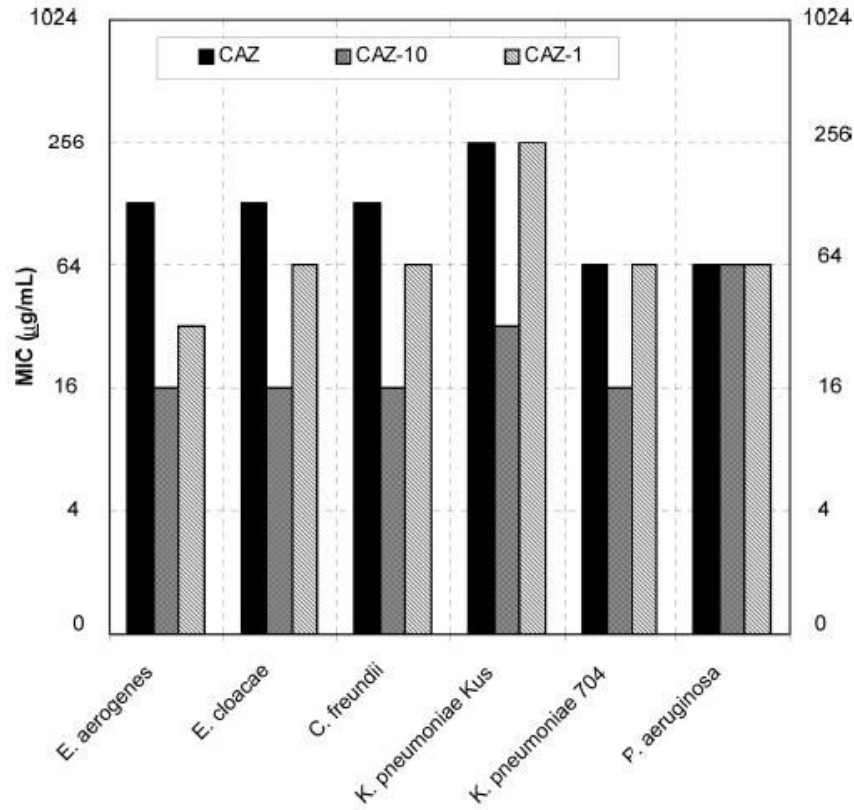

Figure 4.

Activity in cell culture of the lead compound 1 and compound $10 \mathrm{vs}$ bacteria from clinical isolates that express AmpC $\beta$-lactamase. 

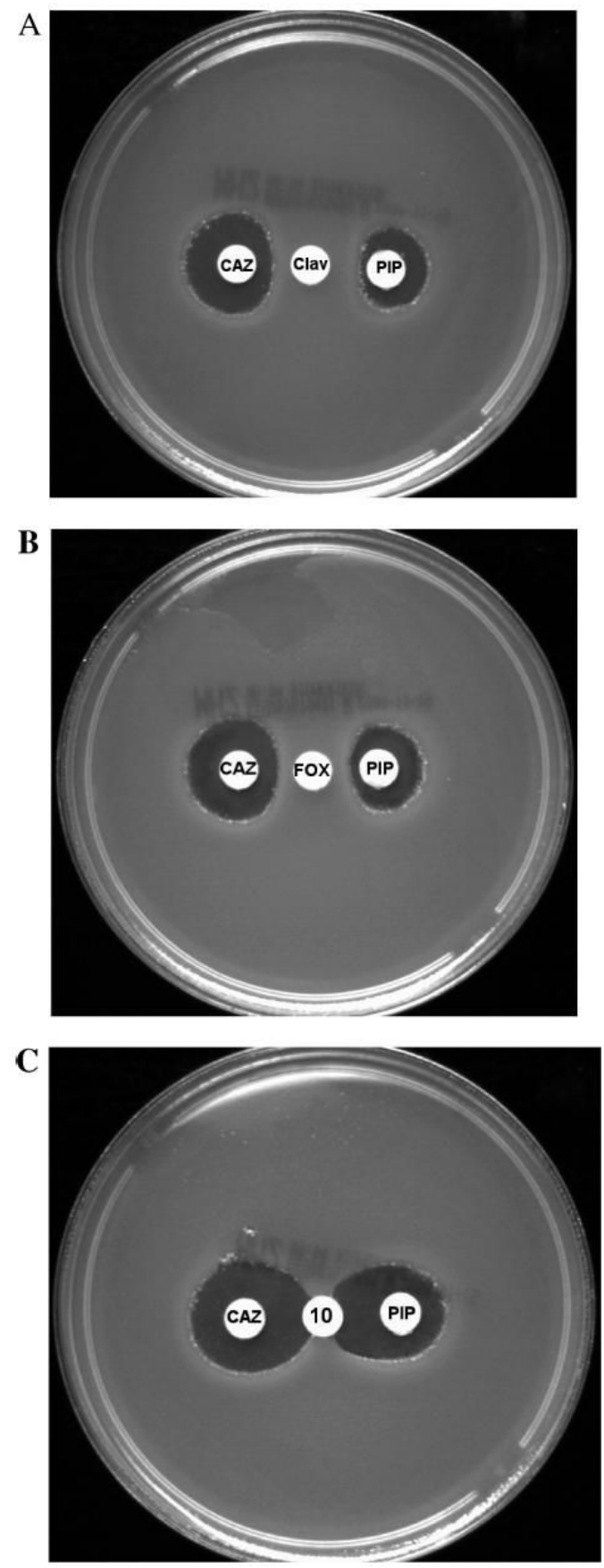

Figure 5.

Effects on up-regulation of a class $C \beta$-lactamase in $E$. cloacae by $\beta$-lactamase inhibitors, each with piperacillin (PIP) and ceftazidime (CAZ): (A) $\beta$-lactam-based inhibitor clavulanate (center); (B) $\beta$-lactam-based inhibitor cefoxitin (FOX); (C) non- $\beta$-lactam inhibitor 10. 


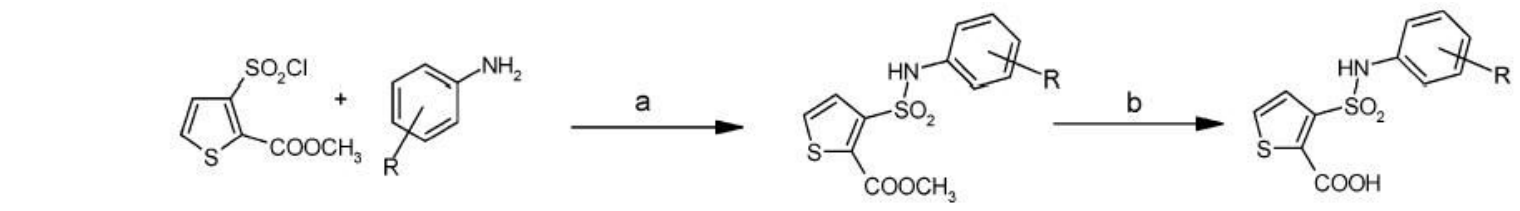

${ }^{a}$ Step a: Py dry, $50{ }^{\circ} \mathrm{C}, \mathrm{N}_{2}$. Step b: $2 \mathrm{~N} \mathrm{NaOH}, 80^{\circ} \mathrm{C}, \mathrm{HCl}$ dil.

Scheme 1.

Synthesis of 3-((4-chloroanilin)sulfonyl)thiophene-2-carboxylic Acid Derivatives ${ }^{\text {a }}$ 


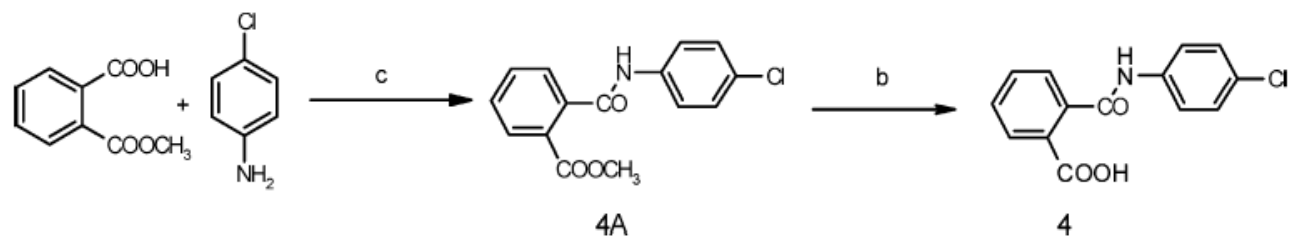

${ }^{a}$ Step c: DMF, DIEA, PyBOP. $60^{\circ} \mathrm{C}, \mathrm{N}_{2}$. Step b: $2 \mathrm{~N} \mathrm{NaOH}, 80^{\circ} \mathrm{C}, \mathrm{HCl}$ dil.

Scheme 2.

Synthesis of N-(4-Chlorophenyl)phthalamic Acid Derivative ${ }^{a}$ 
Table 1

$K_{\mathrm{i}}$ Values of Thiophene-Carboxy Derivatives vs AmpC

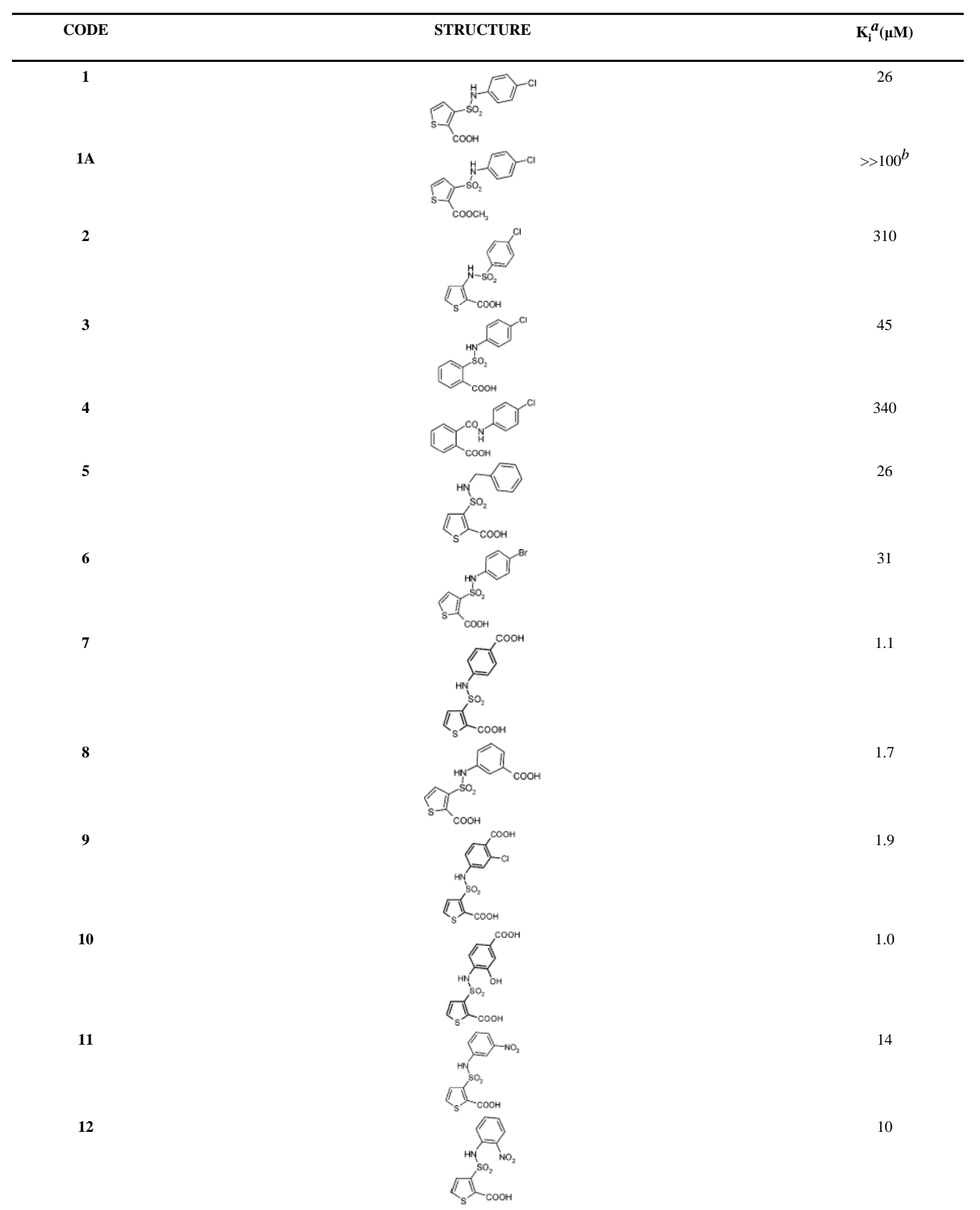




\begin{tabular}{|c|c|c|}
\hline CODE & STRUCTURE & $\mathbf{K}_{\mathrm{i}}^{a}(\mu \mathrm{M})$ \\
\hline 13 & & 8.7 \\
\hline 13A & & 170 \\
\hline 14 & & 250 \\
\hline
\end{tabular}

${ }^{a}$ Estimated error margins on the $K_{\mathrm{i}}$ values are $\pm 50 \%$.

${ }^{b}$ No detectable inhibition at $100 \mu \mathrm{M}$ concentration of compound. 
Table 2

X-ray Data Collection and Refinement Statistics for AmpC/10 and AmpC/11

\begin{tabular}{|c|c|c|}
\hline & AmpC/10 & AmpC/11 \\
\hline \multicolumn{3}{|l|}{ cell constants } \\
\hline$a(\AA)$ & 117.75 & 118.469 \\
\hline$b(\AA)$ & 76.77 & 76.279 \\
\hline$c(\AA)$ & 97.90 & 97.674 \\
\hline$\beta(\operatorname{deg})$ & 116.63 & 116.37 \\
\hline space group & $C_{2}$ & $C_{2}$ \\
\hline resolution $(\AA)$ & 1.97 & 1.96 \\
\hline unique reflcns & 55,165 & 53,587 \\
\hline total obs & 105,106 & 154,202 \\
\hline$R_{\text {merge }}(\%)$ & $9.1(25.8)^{a}$ & $5.4(39.3)^{a}$ \\
\hline completeness $(\%)^{b}$ & $93.7(94.6)$ & $95.4(90.3)$ \\
\hline resolution range for refinement $(\AA)$ & $1.97-20(1.97-2.04)$ & $1.96-20(1.96-2.03)$ \\
\hline$\langle I\rangle /\left\langle\sigma_{\mathrm{I}} ;\right\rangle$ & $9.43(2.14)$ & $11.07(2.09)$ \\
\hline no. of protein residues & 709 & 700 \\
\hline no. of water molecules & 516 & 313 \\
\hline rmsd bond lengths $(\AA)$ & 0.0148 & 0.0085 \\
\hline rmsd bond angles (deg) & 1.7413 & 1.472 \\
\hline$R_{\text {cryst }}(\%)$ & 16.84 & 19.81 \\
\hline$R_{\text {free }}(\%)^{c}$ & 21.53 & 22.65 \\
\hline $\begin{array}{l}\text { av } B \text { factor, protein atoms }\left(\AA^{2} ; \text { molecules } 1 \text { and } 2\right) \\
\text { av } B \text { factor, inhibitor atoms }\left(\AA^{2} ; \text { molecule } 1\right)\end{array}$ & $\begin{array}{l}17.71 \\
39.78\end{array}$ & 28.57 \\
\hline
\end{tabular}

${ }^{a}$ Highest resolution shell in parentheses. Subsequent values in parentheses are for that shell.

${ }^{b}$ Fraction of theoretically possible reflections observed.

${ }^{c} R_{\text {free was calculated with } 5 \% \text { of reflections, randomly selected, set aside. }}$ 
Table 3

Synergy of Compounds 10, 11, and 1 with Ceftazidime against Clinical Strains Producing AmpC-type $\beta$ lactamase

\begin{tabular}{|c|c|c|c|c|}
\hline \multirow[b]{2}{*}{ strains } & \multicolumn{4}{|c|}{$\operatorname{MIC}^{a}(\mu \mathrm{g} / \mathrm{mL})$} \\
\hline & $\mathrm{CAZ}^{b}$ & $\mathrm{CAZ} / 10^{c}$ & $\mathrm{CAZ} / 11^{c}$ & $\mathrm{CAZ} / \mathbf{1}^{c}$ \\
\hline E. aerogenes 298 & 128 & 16 & 64 & 32 \\
\hline E. cloacae $\mathrm{P} 99$ & 128 & 16 & 128 & 64 \\
\hline C. freundii 63 & 128 & 16 & 64 & 64 \\
\hline K. pneumoniae Kus & 256 & 32 & 256 & 256 \\
\hline K. pneumoniae 704 & 64 & 16 & 64 & 64 \\
\hline P. aeruginosa $\mathrm{CF} 34$ & 64 & 64 & 64 & 64 \\
\hline
\end{tabular}

${ }^{a}$ Minimum inhibitory concentration.

${ }^{b} \mathrm{CAZ}=$ ceftazidime.

${ }^{c}$ The inhibitor:CAZ ratio was 1:1, and the concentration of DMSO was always less than $1 \%$. 